\title{
UPDATE
}

\section{Chlamydia trachomatis diagnostics}

\section{A Chernesky}

Nucleic acid amplification (NAA) assays for the diagnosis of Chlamydia trachomatis infections started to appear in the peer reviewed literature about 12 years ago and during that period we have seen an incredible effort put into the development and evaluation of commercially developed NAA kits to diagnose and treat infections.

Correspondence to: M A Chernesky, Pathology and Molecular Medicine, McMaster University, St Joseph's Healthcare, 50 Charlton Avenue East, Hamilton, Ontario,

Canada L8N 4A6

chernesk@mcmaster.ca
$\mathrm{B}$ fore the nucleic acid amplification (NAA) decade laboratories were doing a commendable job using non-NAA assays, especially if you consider that before 1980 very few diagnostic laboratories were in the chlamydial diagnostics business, because of the cell culture and microscopy capabilities required. Some laboratories were performing serology for $C$ trachomatis, which provided questionable clinically useful information. ${ }^{1}$ The major diagnostic breakthrough was the commercialisation of antigen detection tests. ${ }^{23}$ The fact that false positives were generated led to the use of confirmatory testing ${ }^{4}$ and, in retrospect, probably identified as many true positives as culture because, in many cases, culturing was not done well in routine diagnostic laboratories. We now seem to be at a crossroads, where the exceptionally better sensitivity of NAA tests threatens the usefulness of non-NAA assays. These antigen detection tests are still being used in some settings and deserve a re-examination of their role. Recent restructuring of an older enzyme immunoassay (EIA) to have an amplified signal, using recycling enzymes, has produced a more promising EIA called IDEIA PCE. ${ }^{5}$ This assay now deserves further critical evaluation of its use. Also during the antigen detection era of the 1980s we saw the commercialisation of nucleic acid hybridisation (NAH) testing, when the PACE 2 assay from GenProbe became available. ${ }^{6}$ This test also contributed substantially towards our understanding of the natural history of $C$ trachomatis infections, but it too is being threatened with replacement by NAA assays. Why not re-examine the NAH concept before discarding the technology? Digene Corporation has constructed its chlamydia hybrid capture NAH test to incorporate signal amplification and recent evaluations have shown good sensitivity and specificity performance. $^{78}$

At the present time four companies have US FDA approved NAA assays for $C$ trachomatis, usually combined with the capacity to diagnose Neisseria gonorrhoeae infections from the same specimen (table 1). These tests have received stringent approval for use on cervical and urethral swabs and first catch (void) urine, and are being sold worldwide. Many peer reviewed publications have appeared comparing the performance of these assays with culture, ${ }^{910}$ antigen detection, ${ }^{11}$ and with each other. ${ }^{12}{ }^{13}$ The most useful comparisons have used multiple specimens and tests to allow comparisons of the combination of specimen type and test to an expanded reference standard (the infected patient). ${ }^{14} 15$ We now are understanding that multiple testing may yield more accurate results. More studies of this type should enable us to create investigation algorithms which are more cost and patient beneficial. Costs have been addressed for testing larger numbers of specimens through pooling and reflex testing the individual specimens that were placed into a positive pool. This appears to be a cost beneficial approach when prevalence rates are low and large numbers of specimens are being processed daily. $^{16}{ }^{17}$

Analysis of the many publications addressing comparative performance qualities of the commercial NAA tests reveals that there are not many differences in sensitivity and specificity on the

Table 1 Selective characteristics of commercial nucleic acid amplification assays for the diagnosis of Chlamydia trachomatis in cervical or urethral swabs and urine

\begin{tabular}{|c|c|c|c|c|c|c|}
\hline \multirow{2}{*}{$\begin{array}{l}\text { Molecular } \\
\text { method* }\end{array}$} & \multirow[b]{2}{*}{ Company } & \multirow[b]{2}{*}{ Assay } & \multirow{2}{*}{$\begin{array}{l}\text { Maximum } \\
\text { specimens per } \\
\text { run }\end{array}$} & \multicolumn{2}{|l|}{ Time (hours) } & \multirow{2}{*}{$\begin{array}{l}\text { Amplification } \\
\text { control }\end{array}$} \\
\hline & & & & Hands-on & Total & \\
\hline LCR & Abbott & LCx & $40 \dagger$ & 1 to 1.5 & 5.5 to 6.25 & No \\
\hline \multirow[t]{2}{*}{ PCR } & \multirow[t]{2}{*}{ Roche } & Amplicor & 92 & 3 to 3.75 & 6.75 to 8 & Optional \\
\hline & & Cobas Amplicor & $44 \ddagger$ & 2 to 2.5 & 5 to 6 & Optional \\
\hline SDA & Becton Dickinson & ProbeTec ET & 46 & 1 to 1.25 & 3.1 to 3.8 & Yes \\
\hline \multirow[t]{2}{*}{ TMA } & \multirow[t]{2}{*}{ Gen-Probe } & Amp CT & 98 & 2.5 & 5.2 & No \\
\hline & & APTIMA Combo 2 & 98 & 1.6 to 2.25 & 5.3 to 6 & No \\
\hline
\end{tabular}

* Ligase chain reaction (LCR), polymerase chain reaction (PCR), strand displacement amplification (SDA), transcription mediated amplification (TMA). †A full amplification run of 40 specimens will require 2 detection runs on the $L C x$ analyser.

$\ddagger$ An amplification run of 44 would require 2 sets of rings to be placed into the instrument at staggered time intervals. 
specimens for which they are approved. ${ }^{18}$ From the limited number of studies published, all of the NAA tests seem to be impacted by inhibitors in certain types of clinical specimens. We know that these inhibitors are not always the same and vary in prevalence according to the amplification technology. We have learned that these amplification inhibitors may account for sensitivities below $100 \%$ and that some of them disappear by heat, freezing and thawing, dilution, and time. ${ }^{19}$ More studies are needed examining the impact of these manoeuvres on diagnosing and treating infections. Critical evaluation of the reporting of inhibitors of NAA tests requires the use of a $C$ trachomatis spike into the clinical specimen before and after extraction and testing. The spike must also be at a strength near the cut-off of each test because if it is too strong no inhibition will ever be seen and if it is too weak it may be lost in the processing and too many specimens will appear to be inhibitory. At the present time we do not have an appreciation for the impact of the extraction process on the relative proportions of inhibitors and indigenous $C$ trachomatis in a specimen going into the test.

The impact of specimen adequacy on the performance of NAA tests has been reported. ${ }^{20}$ Examination of the specimen by the laboratory before testing it by NAA is a debatable procedure, as inadequate specimens require corrective follow up, requiring patient revisits and a new collection; this can be difficult and unproductive. A simple rapid test for specimen adequacy in the hands of the specimen collector might be an initiative worth developing and evaluating.

Examination of the procedures involved in the commercially available NAA tests are summarised in table 1 and reveal that all of the assays, as they are constructed, suffer from limited throughput. Technician hands-on time varies from approximately 1 hour for the Abbott LCX, and the BD ProbeTec systems, to 3-4 hours for the Roche Amplicor. The total time to get the answer is 5-8 hours for all of the tests, except for the ProbeTec which is 3-4 hours. GenProbe's Aptima Combo and TMA tests and the Abbott LCX do not have an internal control to monitor for amplification inhibitors, whereas Roche's Amplicor has one that is optional. The BD ProbeTec does have an internal control but it is not specific for measuring inhibition of the amplification of $C$ trachomatis DNA (unpublished data).

Choosing one of these assays, which are all priced about the same, tends to be made on customer confidence in the company involved, as performances are similar. Lack of throughput is still a problem for high volume laboratories. The use of pooling and automated pipetting at the front end of these assays has helped, ${ }^{21}$ but industry needs to provide capacity for processing larger numbers of specimens while preserving non-contaminating integrity.

Because $C$ trachomatis infections of the lower genital tract are commonly asymptomatic they often do not get diagnosed and treated. The result of this inefficiency in infection management has been the substantial increase in upper tract infections, especially in younger, sexually active women, leading to alarming increases in rates of pelvic inflammatory disease and the sequelae of infertility, ectopic pregnancy, and chronic pelvic pain. We now have excellent diagnostics that can be used on non-invasive specimens, such as urine, ${ }^{12}$ and swabs from the vagina, ${ }^{22}$ vulva, ${ }^{23}$ and introitus. ${ }^{24}$ We need more studies on the usefulness of these specimens which can be self collected. We need to determine the role of inhibitors of NAA tests for these newer specimens, which in some studies appear to be more sensitive than urine.

Cost-benefit analysis studies have shown that focused screening for $C$ trachomatis by testing non-invasive samples from sexually active women can be effective. ${ }^{25}$ We need more studies examining the feasibility and benefits to women of screening men.
Last, but not least, the commercialisation of diagnostics for $C$ trachomatis and $N$ gonorrhoeae infections has, for the most part, concentrated on the diagnosis and treatment in patients from developed countries. It is now well recognised that the burden of $C$ trachomatis infections is probably even greater in the developing world. ${ }^{27} \mathrm{C}$ trachomatis infections have been implicated to amplify the HIV epidemic in certain settings. ${ }^{28}$ The new focus should be to improve existing technology; and to reformat the best of the technologies into simple, inexpensive point of care tests so that more of the world's population can be screened and treated, and the "silent" epidemic can be brought under control worldwide.

\section{REFERENCES}

1 Bas S, Muzzin P, Ninet B, et al. Chlamydial serology: comparative diagnostic value of immunoblotting, microimmunofluorescence test, and immunoassays using different recombinant proteins as antigens. J Clin Microbiol 2001;39:1368-77.

2 Chernesky M, Castriciano S, Sellors J, et al. Detection of Chlamydia trachomatis antigens in urine as an alternative to swabs and cultures. $J$ Infect Dis 1990;161:124-6.

3 Leonardi GP, Seitz M, Edstrom R, et al. Evaluation of three immunoassays for detection of Chlamydia trachomatis in urine specimens from asymptomatic males. J Clin Microbiol 1992;30:2793-6.

4 Moncada J, Schachter J, Bolan G, et al. Confirmatory assay increases specificity of the Chlamydiazyme test for Chlamydia trachomatis infection of the cervix. J Clin Microbiol 1990;28:1770-3.

5 Chernesky M, Jang D, Copes D, et al. Comparison of a polymer conjugate-enhanced enzyme immunoassay to ligase chain reaction for diagnosis of Chlamydia trachomatis in endocervical swabs. J Clin Microbiol 2001;39:2306-7.

6 Stary A, Teodorowicz L, Hörting-Müller I, et al. Evaluation of the Gen-Probe PACE 2 and the Microtrak enzyme immunoassay for diagnosis of Chlamydia trachomatis in urogenital samples. Sex Transm Dis 1994;21:26-30.

7 Modarress K, Cullen A, Jaffurs W, et al. Detection of Chlamydia trachomatis and Neisseria gonorrhoeae in swab specimens by the Hybrid Capture II and PACE 2 nucleic acid probe tests. Sex Transm Dis 1999;26:303-8.

8 Schachter J, Hook III EW, McCormack WM, et al. Ability of the Digene Hybrid Capture II test to identify Chlamydia trachomatis and Neisseria gonorrhoeae in cervical specimens. J Clin Microbiol 1999; 37:3668-71

9 Schachter J, Stamm WE, Quinn TC, et al. Ligase chain reaction to detect Chlamydia trachomatis infection of the cervix. J Clin Microbiol 1994:32:2540-3.

10 Bauwens JE, Clark AM, Stamm WE. Diagnosis of Chlamydia trachomatis endocervical infections by a commercial polymerase chain reaction assay. J Clin Microbiol 1993;31:3023-7.

11 Van Dyck E, leven M, Pattyn S. Detection of Chlamydia trachomatis and Neisseria gonorrhoeae by enzyme immunoassay, culture, and three nucleic acid amplification tests. J Clin Microbiol 2001;39:1751-6.

12 Chernesky M, Jang, D, Lee $H$, et al. Diagnosis of Chlamydia trachomatis infections in men and women by testing first-void urine by ligase chain reaction. J Clin Microbiol 1994:32:2682-5.

13 Puolakkainen M, Hiltunen-Back E, Reunala T, et al. Comparison of performances of two commercially available tests, a PCR assay and a ligase chain reaction test, in detection of urogenital Chlamydia trachomatis infection. J Clin Microbiol 1998;36:1489-93.

14 Van der Pol B, Ferrero D, Buck-Barrington L, et al. Multicenter evaluation of the BDProbeTec ET system for detection of Chlamydia trachomatis and Neisseria gonorrhoeae in urine specimens, female endocervical swabs, and male urethral swabs. J Clin Microbiol 2001;39:1008-16.

15 Thejls H, Gnarpe J, Gnarpe H, et al. Expanded gold standard in the diagnosis of Chlamydia trachomatis in a low prevalence population: diagnostic efficacy of tissue culture, direct immunofluorescence, enzyme immunoassay, PCR and serology. Genitourin Med 1994;70: 300-3

16 Kacena KA, Quinn SB, Howell MR, et al. Pooling urine samples for ligase chain reaction screening for genital Chlamydia trachomatis infection in asymptomatic women. J Clin Microbiol 1998;36:481-5.

17 Kapala J, Copes D, Sproston A, et al. Pooling cervical swabs and testing by ligase chain reaction accurate and cost-saving strategies for diagnosis of Chlamydia trachomatis. J Clin Microbiol 2000;38:2480-3.

18 Black, CM. Current methods of laboratory diagnosis of Chlamydia trachomatis infections. Clin Microbiol Rev 1997;10:160-84.

19 Mahony J, Chong S, Jang D, et al. Urine specimens from pregnant and nonpregnant women inhibitory to amplification of Chlamydia trachomatis nucleic acid by PCR, ligase chain reaction, and transcription-mediated amplification: identification of urinary substances associated with inhibition and removal of inhibitory activity. J Clin Microbiol 1998; 36:3122-6.

20 Kellogg JA, Seiple JW, Klinedinst JL, et al. Diff-quick stain as a simplified alternative to Papanicolaou stain for determination of quality of endocervical specimens submitted for PCR detection of Chlamydia trachomatis. J Clin Microbiol 1996;34:2590-2. 
21 Hanson KL, Cartwright CP. Evaluation of an automated liquid-handling system (Tecan Genesis RSP 100) in the Abbott LCx assay for Chlamydia trachomatis. J Clin Microbiol 2001;39:1975-7.

22 Stary A, Schuh E, Kerschbaumer M, et al. Performance of transcription-mediated amplification and ligase chain reaction assays for detection of chlamydial infection in urogenital samples obtained by invasive and noninvasive methods. J Clin Microbiol 1998;36:2666-70.

23 Stary A, Najim B, Lee HH. Vulval swabs as alternative specimens for ligase chain reaction detection of genital chlamydial infection in women. J Clin Microbiol 1997:35:836-8.

24 Wiesenfeld HC, Rideout A, Macio I, et al. The vaginal introitus: a novel site for Chlamydia trachomatis testing in women. Am J Obstet Gynecol $1996 ; 174: 1542-6$
25 Howell MR, Quinn TC, Gaydos CA. Screening for Chlamydia trachomatis in asymptomatic women attending family planning clinics-a trachomatis in asymptomatic women attending tamily plannin cost-effectiveness an
$1998 \cdot 128: 277-84$

26 Marrazzo JM, Celum CL, Hillis S, et al. Performance and cost-effectiveness of selective screening criteria for Chlamydia trachomatis infection in women (implications for a National Chlamydia Control Strategy). Sex Transm Dis 1997;24:131-41.

27 Paxton LA, Sewankambo N, Gray R, et al. Asymptomatic non-ulcerative genital tract infections in a rural Ugandan population. Sex Transm Dis 1998:74:42 1-5.

28 Laga M, Manoka A, Kivuvu M, et al. Non-ulcerative sexually transmitted diseases as risk factors for HIV-1 transmission in women: results from a cohort study. AIDS 1993;7:95-102.

\section{NEW STI ONLINE SUBMISSION AND REVIEW SYSTEM}

I am pleased to inform authors and reviewers that as of 21 February 2002, STI will be using a new online submission and review system. Developed by Highwire Press (CA, USA), Bench>Press is a fully integrated electronic system which utilises the web to allow rapid and efficient submission of manuscripts. It also allows the peer review process to be conducted entirely online. The aim, apart from saving trees, is to speed up the frequently frustrating progress from submission to publication.

Authors can submit their manuscript in any standard word processing software. Standard graphic formats acceptable are: .jpg, .tiff, .gif, and eps. (nb. multipage powerpoint files are NOT acceptable). The text and graphic files are automatically converted to PDF for ease of distribution and reviewing purposes. Authors are asked to approve their submission before it formally enters the reviewing process. On approval by the authors, the submission is passed to the editor and/or reviewers via the web. All transactions are secure.

To access the system click on "SUBMIT YOUR MANUSCRIPT HERE" on the STI homepage: http://www.sextransinf.com/ or you can access Bench>Press directly at http://submit-sti.bmijournals.com/.

We are very excited with this new development and I would encourage authors and reviewers to use the online system where possible. It really is simple to use and should be a big improvement on the current peer review process. Full instructions can be found on Bench>Press and STI online. Please contact Natalie Davies, Project Manager, ndavies@bmigroup.com for further information.

\section{PRE-REGISTER WITH THE SYSTEM}

We would be grateful if all Sexually Transmitted Infections authors and reviewers pre-registered with the system. This will give you the opportunity to update your contact and expertise data, allowing us to provide you with a more efficient service.

\section{Instructions for registering}

1. Enter http://submit-sti.bmijournals.com

2. Click on "Create a New Account" in the upper left hand side of the Bench>Press homepage

3. Enter your email address in the space provided.

4. Choose a password for yourself and enter it in the spaces provided.

5. Complete the question of your choice to be used in the event you cannot remember your password at a later time.

6. Click on the "Save" button at the bottom of the screen.

7. Check the email account you registered under. An email will be sent to you with a verification number and URL.

8. Once you receive this verification number, click on the URL hyperlink and enter the verification number in the relevant field. This is for security reasons and to check that your account is not being used fraudulently.

9. Enter/amend your contact information, and update your expertise data. 